\title{
Nonlinear Dynamics of Two-State Quantum Dot Lasers under Optical Feedback
}

\author{
Xiang-Hui Wang ${ }^{1}$, Zheng-Mao Wu ${ }^{1}{ }^{\oplus}$, Zai-Fu Jiang ${ }^{1,2}$ and Guang-Qiong Xia ${ }^{1, *(1)}$ \\ 1 Chongqing City Key Laboratory of Micro \& Nano Structure Optoelectronics, \\ School of Physical Science and Technology, Southwest University, Chongqing 400715, China; \\ wangxianghui@email.swu.edu.cn (X.-H.W.); zmwu@swu.edu.cn (Z.-M.W.); \\ jzf23003@email.swu.edu.cn (Z.-F.J.) \\ 2 School of Mathematics and Physics, Jingchu University of Technology, Jingmen 448000, China \\ * Correspondence: gqxia@swu.edu.cn
}

check for updates

Citation: Wang, X.-H.; Wu, Z.-M.; Jiang, Z.-F.; Xia, G.-Q. Nonlinear Dynamics of Two-State Quantum Dot Lasers under Optical Feedback. Photonics 2021, 8, 300. https:// doi.org/10.3390/photonics 8080300

Received: 17 May 2021

Accepted: 23 July 2021

Published: 27 July 2021

Publisher's Note: MDPI stays neutral with regard to jurisdictional claims in published maps and institutional affiliations.

Copyright: (c) 2021 by the authors. Licensee MDPI, Basel, Switzerland. This article is an open access article distributed under the terms and conditions of the Creative Commons Attribution (CC BY) license (https:// creativecommons.org/licenses/by/ $4.0 /)$.

\begin{abstract}
A modified rate equation model was presented to theoretically investigate the nonlinear dynamics of solitary two-state quantum dot lasers (TSQDLs) under optical feedback. The simulated results showed that, for a TSQDL biased at a relatively high current, the ground-state (GS) and excited-state (ES) lasing of the TSQDL can be stimulated simultaneously. After introducing optical feedback, both GS lasing and ES lasing can exhibit rich nonlinear dynamic states including steady state (S), period one (P1), period two (P2), multi-period (MP), and chaotic (C) state under different feedback strength and phase offset, respectively, and the dynamic states for the two lasing types are always identical. Furthermore, the influences of the linewidth enhancement factor (LEF) on the nonlinear dynamical state distribution of TSQDLs in the parameter space of feedback strength and phase offset were also analyzed. For a TSQDL with a larger LEF, much more dynamical states can be observed, and the parameter regions for two lasing types operating at chaotic state are widened after introducing optical feedback.
\end{abstract}

Keywords: nonlinear dynamics; quantum dot lasers; optical feedback; chaotic; linewidth enhancement factor (LEF)

\section{Introduction}

After introducing external perturbations, semiconductor lasers (SLs) can exhibit rich nonlinear dynamics [1,2], which can be applied in many fields such as random number generation, secure communication, photonic microwave signal generation, all-optical logic gates, and reservoir computing [3-7].

Quantum dot (QD) lasers are self-assembled nanostructured SLs. Compared with traditional quantum well (QW) SLs, QD lasers have many advantages such as low threshold current density [8], high temperature stability [9], low chirp [10], and large modulation bandwidth [11]. Such unique characteristics make QD lasers become excellent candidate light sources in optical communication, optical interconnection, silicon photonic integrated circuits, and photonic microwave generation, etc. [12-16]. Due to strong three-dimension quantum confinement of the carriers, QD lasers have discrete energy levels and state densities, which lead to their unique emission performances. Related studies have shown that there exist two current thresholds in ordinary QD lasers. When the bias current is increased to the first threshold, QD lasers can emit on the ground-state (GS). Continuously increasing the bias current, the number of carriers at the excited-state (ES) increases rapidly. Once the bias current exceeds a certain value (the second threshold), QD lasers can simultaneously emit on GS and ES. Correspondingly, such QD lasers are named as two-state QD lasers (TSQDLs) [17,18]. Via some technologies, QD lasers can emit solely on GS or ES, and the corresponding QD lasers are named as GS-QD lasers and ES-QD lasers, respectively [19,20]. 
Previous studies have shown that different types of QD lasers can exhibit different performances. GS-QD lasers possess a low threshold current and low sensitivity to optical feedback owing to relatively low energy levels and strong damping of relaxation oscillation [21,22]. Compared with GS-QD lasers, ES-QD lasers possess larger modulation bandwidths and richer nonlinear dynamics under external perturbations owing to faster carrier capture rates [23-26]. Different from GS-QD lasers and ES-QD lasers, TSQDLs can lase at two wavelengths separated by several tens of nanometers [27] and exhibit lower intensity noise [28], which can be applied in many fields such as terahertz (THz) signal generation, two-color light sources, two color mode-locking, all-optical processing, and artificial optical neurons, etc. [29-32]. In recent years, the investigations on the nonlinear dynamics of TSQDLs under external perturbations have attracted special attention. Through introducing optical injection into GS, the ES emission in TSQDLs can be suppressed and the mode switching from ES to GS is triggered [33,34]. Through scanning the optical power of injection light along different varying routes, a bistable phenomenon can be observed $[35,36]$. After introducing optical feedback to TSQDLs, many interesting phenomena can be observed such as mode switching and mode competition between the GS and ES [37,38], energy exchanging among longitudinal modes [39], two-color oscillating [40], and anti-phase low frequency fluctuating [41]. However, to our knowledge, the nonlinear dynamical state evolution of TSQDLs under optical feedback has not been reported.

In this work, based on three-level model of QD lasers [42,43], a modified theoretical model for TSQDLs under optical feedback was presented to numerically investigate the nonlinear dynamical characteristics of TSQDLs under optical feedback. Moreover, the influences of the linewidth enhancement factor (LEF) on the nonlinear dynamical state distribution of TSQDLs in the parameter space of feedback strength and phase offset were also analyzed.

\section{Rate Equation Model}

The theoretical model in this work was based on the three-level model of QD lasers, which has been adopted to analyze the static and dynamic behaviors, noise characteristics of QD lasers operating at free-running [42,43], and the small-signal modulation response and relative intensity noise of QD lasers under optical injection-locking conditions [44]. Figure 1 shows the simplified schematic diagram of the carrier dynamics for two-state QD lasers (TSQDLs) based on the three-level model [45]. In this system, two relatively low energy levels involving ground state (GS) and the first excited state (ES) were taken into account. The electrons and holes were treated as neutral excitons (electron-hole pairs), and the stimulated emission can occur in GS and ES. It was assumed that all QDs had the same size and the active region consisted of only one QD ensemble. Therefore, the inhomogeneous broadening effect was ignored. As shown in the figure, the carriers were injected directly into the wetting layer (WL) from the electrodes. In the $\mathrm{WL}$, owing to Auger recombination and phonon-assisted scattering processes [46,47], some carriers were captured into ES with a captured time $\tau_{E S}^{W L}$. Some carriers relaxed directly into GS with a relaxation time $\tau_{G S}^{W L}$. The rest of the carriers recombined spontaneously with a time $\tau_{W L}^{\text {spon }}$. For the carriers in ES, some of them relaxed into GS with a relaxation time $\tau_{G S}^{E S}$ and the other carriers recombined spontaneously with an emission time $\tau_{E S}^{\text {spon }}$. On the other hand, owing to the thermal excitation effect, some carriers were excited into WL with an escape time $\tau_{W L}^{E S}$. Similarly, the carriers in GS were excited into ES with an escape time $\tau_{E S}^{G S}$, and some carriers also recombined spontaneously with an emission time $\tau_{G S}^{\text {spon }}$. Based on the three-level model, after referring to the optical feedback processing methods in Ref. [48], we propose modified rate equations for describing the nonlinear dynamics of TSQDLs under optical feedback as follows:

$$
\frac{d N_{W L}}{d t}=\frac{\eta I}{q}+\frac{N_{E S}}{\tau_{W L}^{E S}}-\frac{N_{W L}}{\tau_{E S}^{W L}}\left(1-\rho_{E S}\right)-\frac{N_{W L}}{\tau_{G S}^{W L}}\left(1-\rho_{G S}\right)-\frac{N_{W L}}{\tau_{W L}^{s p o n}}
$$




$$
\begin{gathered}
\frac{d N_{E S}}{d t}=\frac{N_{W L}}{\tau_{E S}^{W L}}\left(1-\rho_{E S}\right)+\frac{N_{G S}}{\tau_{E S}^{G S}}\left(1-\rho_{E S}\right)-\frac{N_{E S}}{\tau_{W L}^{E S}}-\frac{N_{E S}}{\tau_{G S}^{E S}}\left(1-\rho_{G S}\right)-\frac{N_{E S}}{\tau_{E S}^{s p o n}}-\Gamma_{P} v_{g} g_{E S} S_{E S} \\
\frac{d N_{G S}}{d t}=\frac{N_{W L}}{\tau_{G S}^{W L}}\left(1-\rho_{G S}\right)+\frac{N_{E S}}{\tau_{G S}^{E S}}\left(1-\rho_{G S}\right)-\frac{N_{G S}}{\tau_{E S}^{G S}}\left(1-\rho_{E S}\right)-\frac{N_{G S}}{\tau_{G S}^{s p o n}}-\Gamma_{P} v_{g} g_{G S} S_{G S} \\
\frac{d S_{G S}}{d t}=\left(\Gamma_{P} v_{g} g_{G S}-\frac{1}{\tau_{p}}\right) S_{G S}+\beta_{s p} \frac{N_{G S}}{\tau_{G S}^{s p o n}}+2 \frac{k}{\tau_{i n}} \sqrt{S_{G S}(t) S_{G S}(t-\tau)} \cos \left(\Delta \phi_{G S}\right) \\
\frac{d S_{E S}}{d t}=\left(\Gamma_{P} v_{g} g_{E S}-\frac{1}{\tau_{p}}\right) S_{E S}+\beta_{s p} \frac{N_{E S}}{\tau_{E S}^{s p o n}}+2 \frac{k}{\tau_{i n}} \sqrt{S_{E S}(t) S_{E S}(t-\tau)} \cos \left(\Delta \phi_{E S}\right) \\
\quad \frac{d \phi_{G S}}{d t}=\frac{\alpha}{2}\left(\Gamma_{P} v_{g} g_{G S}-\frac{1}{\tau_{p}}\right)-\frac{k}{\tau_{i n}} \sqrt{\frac{S_{G S}(t-\tau)}{S_{G S}(t)}} \sin \left(\Delta \phi_{G S}\right) \\
\frac{d \phi_{E S}}{d t}=\frac{\alpha}{2}\left(\Gamma_{P} v_{g} g_{E S}-\frac{1}{\tau_{p}}\right)-\frac{k}{\tau_{i n}} \sqrt{\frac{S_{E S}(t-\tau)}{S_{E S}(t)}} \sin \left(\Delta \phi_{E S}\right)
\end{gathered}
$$

where $W L, E S, G S$ are the wetting layer, excited-state, and ground-state, respectively, and the superscript spon represents the spontaneous emission. $N, S, \phi$ are the carrier number, photon number, and phase, respectively. $I$ is the injection current, $\eta$ is the current injection efficiency, and $q$ is the electron charge. $\Gamma_{p}$ is the optical confinement factor, $v_{g}\left(=c / n_{r}\right.$, where $c$ is the light speed in vacuum and $n_{r}$ the refractive index) is the group velocity. $\tau_{p}$ is the photon lifetime, $\tau_{\text {in }}$ is the round-trip time in the laser cavity, and $\tau\left(=2 l_{e x} / c\right.$, where $l_{e x}$ the external cavity length) is the round-trip time of external cavity. $k$ is the feedback strength, and $\alpha$ is the linewidth enhancement factor. Considering that GS and ES have twofold degeneration and fourfold degeneration, respectively, the carrier occupation probabilities and the gains of GS and ES can be expressed as [42]:

$$
\begin{gathered}
\rho_{G S}=\frac{N_{G S}}{2 N_{B}} ; \rho_{E S}=\frac{N_{E S}}{4 N_{B}} \\
g_{G S}=\frac{a_{G S}}{1+\xi_{G S} \frac{S_{G S}}{V_{S}}} \frac{N_{B}}{V_{B}}\left(2 \rho_{G S}-1\right) \\
g_{E S}=\frac{a_{E S}}{1+\xi_{E S} \frac{S_{E S}}{V_{S}}} \frac{N_{B}}{V_{B}}\left(2 \rho_{E S}-1\right)
\end{gathered}
$$

where $N_{B}$ is the number of quantum dots. $a_{G S}$ and $a_{E S}$ are the differential gain, $\xi_{G S}$ and $\xi_{E S}$ are the gain compression factor, vs. is the volume of the laser field inside the cavity, and $V_{B}$ is the volume of the active region. The feedback phase variation can be described as:

$$
\begin{gathered}
\Delta \phi_{G S}=\phi_{G S}(t)-\phi_{G S}(t-\tau)+\omega_{G S} \tau \\
\Delta \phi_{E S}=\phi_{E S}(t)-\phi_{E S}(t-\tau)+\omega_{E S} \tau
\end{gathered}
$$

where $\omega_{G S}$ and $\omega_{E S}$ are the angular frequencies for GS and ES lasing, respectively.

The rate equations can be numerically solved by the fourth-order Runge-Kutta method via MATLAB software. During the calculations, the used parameters and their values are given in Table 1 [42]: 


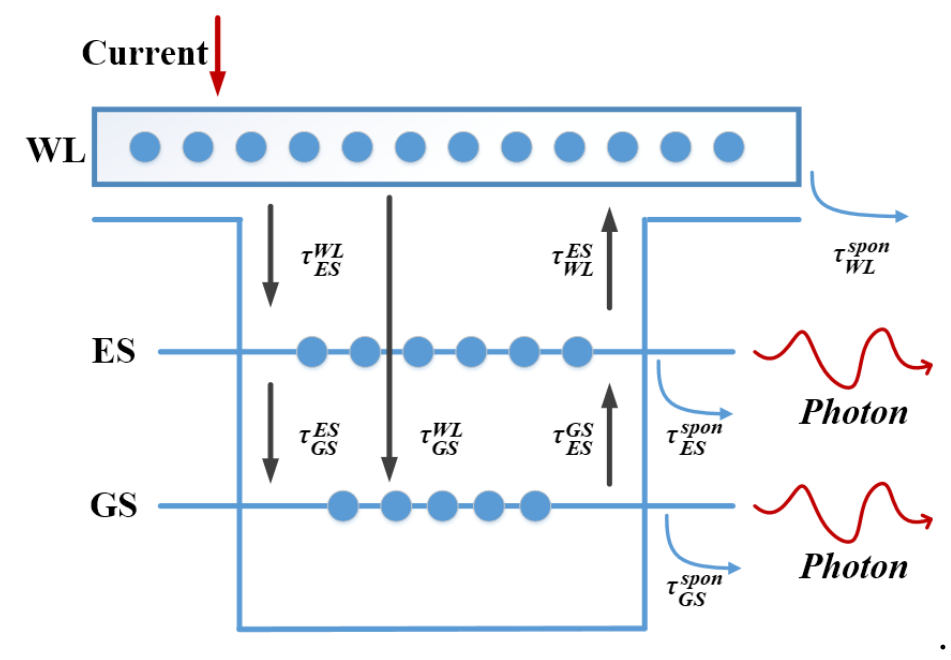

Figure 1. Schematic diagram of the carrier dynamics for QD lasers based on the three-level model. WL: wetting layer; GS: ground state; ES: excited state.

Table 1. Simulation parameters of the QD lasers.

\begin{tabular}{|c|c|c|}
\hline Symbol & Parameter & Value \\
\hline$\tau_{E S}^{W L}$ & Capture time from WL to ES & $12.6 \mathrm{ps}$ \\
\hline$\tau_{G S}^{E S}$ & Capture time from ES to GS & $8 \mathrm{ps}$ \\
\hline$\tau_{G S}^{W L}$ & Relaxation time from WL to GS & $15 \mathrm{ps}$ \\
\hline$\tau_{E S}^{G S}$ & Escape time from GS to ES & $10.4 \mathrm{ps}$ \\
\hline$\tau_{W L}^{E S}$ & Escape time from ES to WL & $5.4 \mathrm{~ns}$ \\
\hline$\tau_{W L}^{\text {spon }}$ & Spontaneous emission time from $W L$ & $0.5 \mathrm{~ns}$ \\
\hline$\tau_{E S}^{\text {spon }}$ & Spontaneous emission time from ES & $0.5 \mathrm{~ns}$ \\
\hline$\tau_{G S}^{\text {spon }}$ & Spontaneous emission time from GS & $1.2 \mathrm{ps}$ \\
\hline$\tau_{P}$ & Photon lifetime & $4.1 \mathrm{ps}$ \\
\hline$N_{B}$ & Total number of QD & $1.0 \times 10^{7}$ \\
\hline$\Gamma_{p}$ & Optical confinement factor & 0.06 \\
\hline$n_{r}$ & Refractive index & 3.5 \\
\hline$\tau_{\text {in }}$ & Round-trip time & $10 \mathrm{ps}$ \\
\hline$a_{G S}$ & Differential gain from GS & $5.0 \times 10^{-15} \mathrm{~cm}^{2}$ \\
\hline$a_{E S}$ & Differential gain from ES & $10.0 \times 10^{-15} \mathrm{~cm}^{2}$ \\
\hline$\xi_{G S}$ & Gain compression factor from GS & $1.0 \times 10^{-16} \mathrm{~cm}^{3}$ \\
\hline$\xi_{E S}$ & Gain compression factor from ES & $8.0 \times 10^{-16} \mathrm{~cm}^{3}$ \\
\hline$\beta_{s p}$ & Spontaneous emission factor & $5.0 \times 10^{-6}$ \\
\hline$\omega_{G S}$ & Angular frequency from GS & $1.446 \times 10^{15} \mathrm{rad} / \mathrm{s}$ \\
\hline$\omega_{E S}$ & Angular frequency from ES & $1.529 \times 10^{15} \mathrm{rad} / \mathrm{s}$ \\
\hline$V_{B}$ & Active region volume & $5.0 \times 10^{-11} \mathrm{~cm}^{3}$ \\
\hline$V_{S}$ & Resonant cavity volume & $0.833 \times 10^{-15} \mathrm{~cm}^{3}$ \\
\hline$\eta$ & Injection efficiency & 0.25 \\
\hline$q$ & Elementary charge & $1.6 \times 10^{-19} \mathrm{C}$ \\
\hline$\tau$ & Feedback delay time & $100 \mathrm{ps}$ \\
\hline$\alpha$ & Linewidth enhancement factor & 3.5 \\
\hline
\end{tabular}

\section{Results and Discussion}

Figure 2 shows the normalized output power of the GS and ES lasing as a function of the injection current for a TSQDL under free-running (solid lines) or optical feedback with 
a feedback strength of $k=0.11$ (dotted lines). For the TSQDL operating at free-running, the threshold currents of the GS and ES lasing were $36 \mathrm{~mA}\left(I_{t h}^{G S}\right)$ and $88 \mathrm{~mA}\left(I_{t h}^{E S}\right)$, respectively. With the increase of the current from $36 \mathrm{~mA}$ to $88 \mathrm{~mA}$, the power of GS lasing gradually increased while the ES lasing was always in a suppressed state. However, once the injection current was exceeded $88 \mathrm{~mA}$, the ES lasing could be observed. Further increasing the current, the power of the ES lasing rapidly increased while the power of the GS lasing increased slowly. Above results are in agreement with those reported in Ref. [43]. After introducing an optical feedback of $k=0.11$, the threshold current for GS slightly decreased, which is similar with that observed in a single-mode distributed feedback semiconductor laser under optical feedback. However, optical feedback raises the threshold of ES. The reason is that the predominant component in the feedback light is originating from GS lasing, and therefore the optical feedback enhances the competitiveness of the GS lasing. Correspondingly, a higher current is needed for ES to start oscillation. In the following, we fixed the current of the TSQDL at $120 \mathrm{~mA}$, at which the power of GS lasing was more than that of ES lasing.

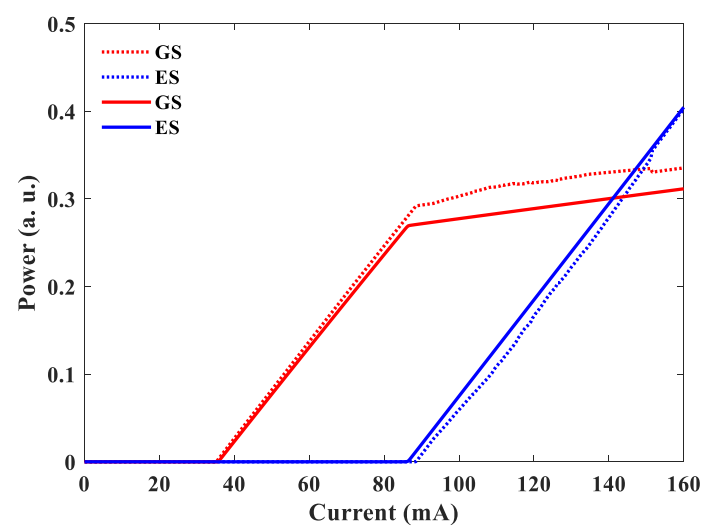

Figure 2. Normalized output power as a function of the injection current for a TSQDL under free-running (solid lines) or optical feedback with a feedback strength of $k=0.11$ (dotted lines).

Figure 3 displays the time series, power spectra, and phase portraits of typical dynamic state output from GS lasing and ES lasing of a TSQDL biased at $120 \mathrm{~mA}$ under optical feedback with $\tau=100$ ps and different $k$. For $k=0.03$, the output intensity of GS lasing (Figure 3(a1)) was nearly a constant, the power spectrum was relatively smooth (Figure 3(a2)), and the phase portrait was a dot (Figure 3(a3)). Obviously, under this case, the dynamical state of GS lasing is a stable (S) state. For $k=0.07$, the time series of GS lasing (Figure 3(b1)) exhibited a stable periodic oscillation with a fundamental frequency of about $6.3 \mathrm{GHz}$ obtained from the power spectrum (Figure 3(b2)), and the phase portrait is a dense dot (Figure 3(b3)). Based on these characteristics, the dynamic state of GS lasing can be judged as a period-one (P1) state. For $k=0.092$, the time series of GS lasing (Figure 3(c1)) behaves periodic oscillation with two peak intensities, both the subharmonic frequency (about $3.1 \mathrm{GHz}$ ) and the fundamental frequency (about $6.3 \mathrm{GHz}$ ) present clearly in the power spectrum (Figure 3(c2)), and the corresponding phase portrait (Figure 3(c3)) is two closed circles, which are typical characteristics of period-two (P2) state. For $k=0.097$, the time series of GS lasing (Figure 3(d1)) exhibited multiple different peaks, a quarter-harmonic frequency component appeared in the power spectrum (Figure 3(d2)), and the phase portrait (Figure 3(d3)) showed multiple loops. These features mean that the dynamical state of GS lasing is a multi-period (MP) state. For $k=0.154$, the time series of GS lasing (Figure 3(e1)) showed a disordered oscillation, and the power spectra were broadened (Figure 3(e2)). In addition, the corresponding phase portrait (Figure 3(e3)) showed a strange attractor. Therefore, the dynamic state of GS lasing can be determined to be the chaotic (C) state. Through comparing the characteristics of ES lasing with those of 
GS lasing, it can be seen that the dynamical states of ES lasing are always the same as those of GS lasing.
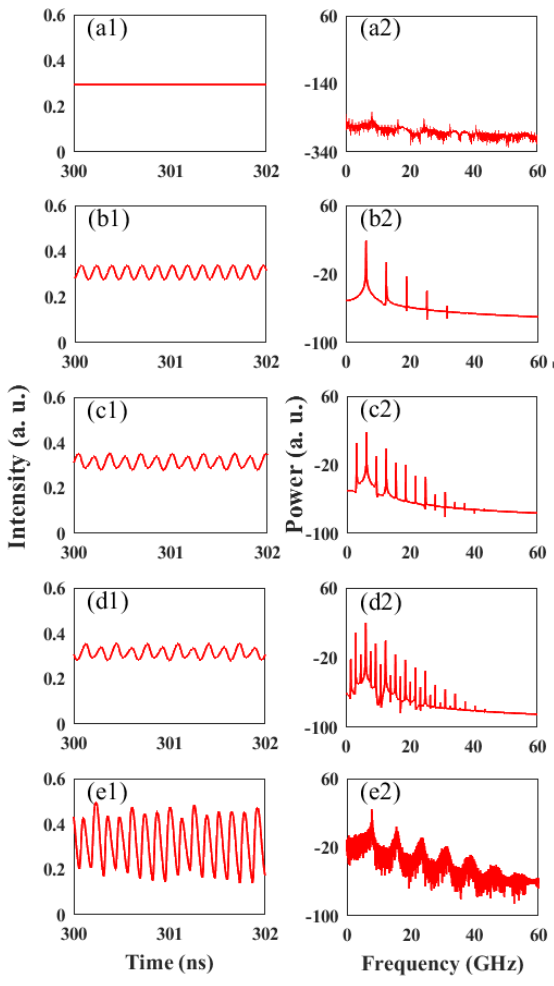
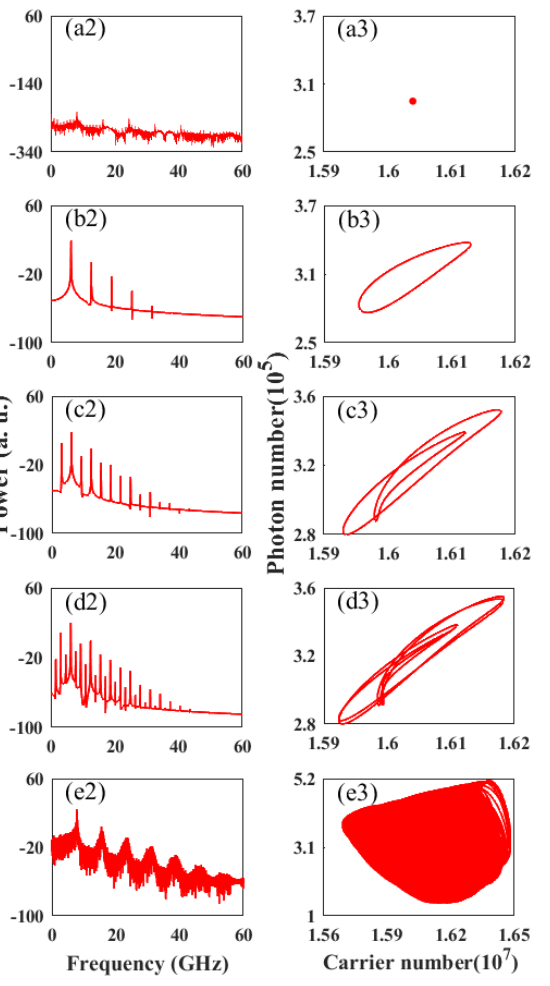
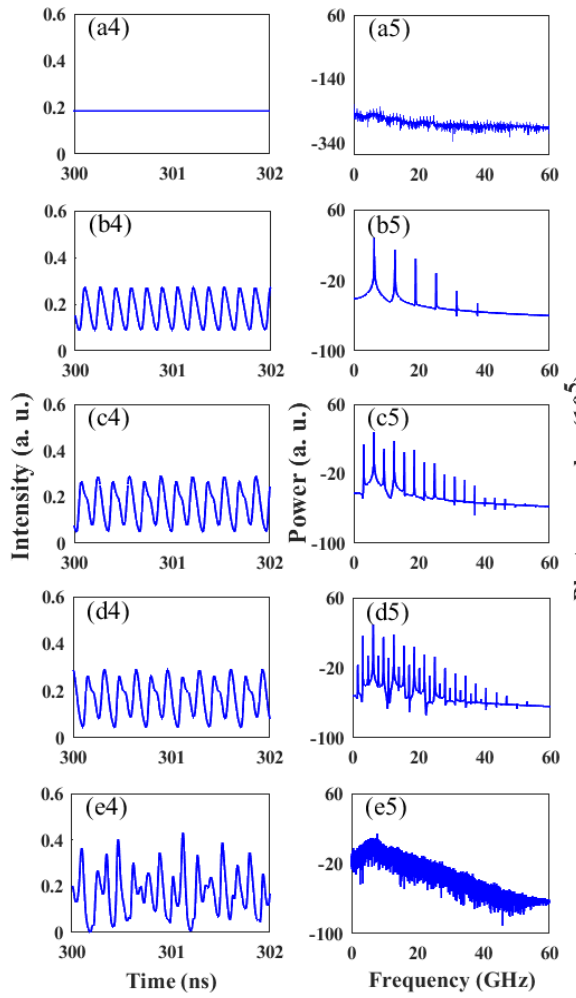
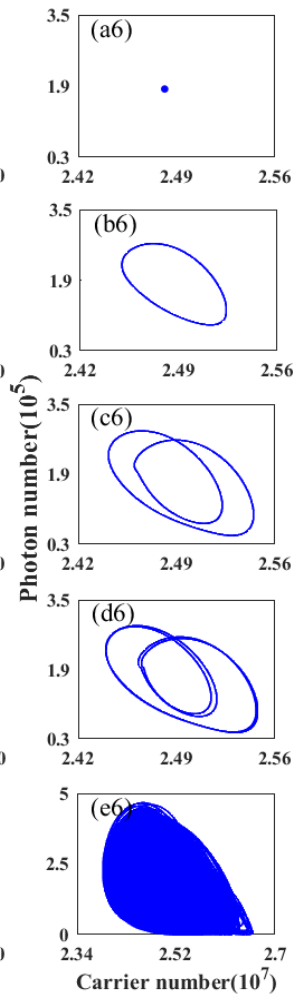

Figure 3. Time series, power spectra, and phase portraits output from GS lasing (red) and ES lasing (blue) in a TSQDL biased at $120 \mathrm{~mA}$ under optical feedback with $\tau=100 \mathrm{ps}$ and $k=0.03(\mathbf{a}), 0.07(\mathbf{b}), 0.092(\mathbf{c}), 0.097(\mathbf{d})$, and $0.154(\mathbf{e})$, respectively.

Above results show that, through setting feedback parameters at different values, some typical dynamical states can be observed for both ES and GS lasing. In order to inspect the evolution route of dynamical state with the feedback strength, Figure 4 presents the bifurcation diagrams of the power extreme and largest Lyapunov exponent (LLE) of the GS lasing and ES lasing as a function of feedback strength. LLE is an important indicator to measure the stability of a laser nonlinear dynamical system [49]. A positive LLE value means that the laser operates at a chaotic state while a negative LLE value corresponds to a steady state. For a laser operating at periodic states, the LLE value tends to approach zero. From this diagram, it can be seen that, with the increase of $k$ from 0 to 0.043 , the output of GS lasing and ES lasing remains in a stable state due to the relatively low feedback strength. Further increasing the feedback strength, the external cavity modes compete with the intrinsic oscillation frequency of the laser, and the dynamic states of GS lasing and ES lasing transform into periodic states including P1, P2, and MP. When the feedback strength exceeds 0.11 , the TSQDL enters into the $C$ state due to coherent collapse. As a result, the dynamics evolution routes of S-P1-P2-MP-C of the GS lasing and ES lasing are presented. Continuously increasing the feedback strength, the laser enters into the chaos state through period-doubling bifurcation, and such an evolution process repeats continuously. 


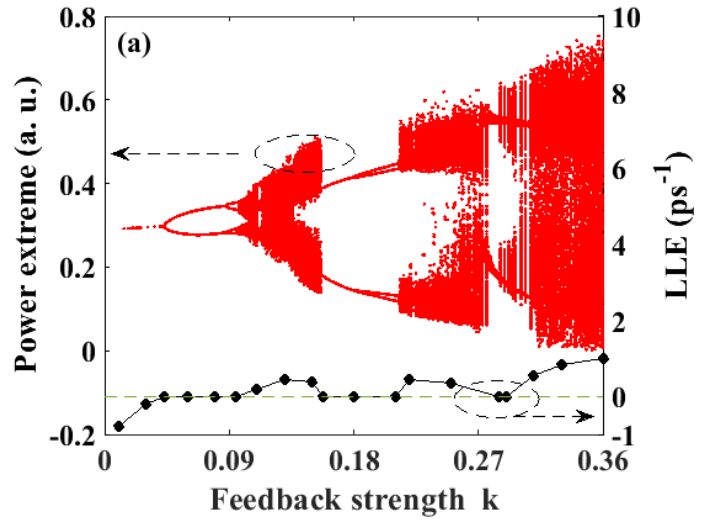

(a)

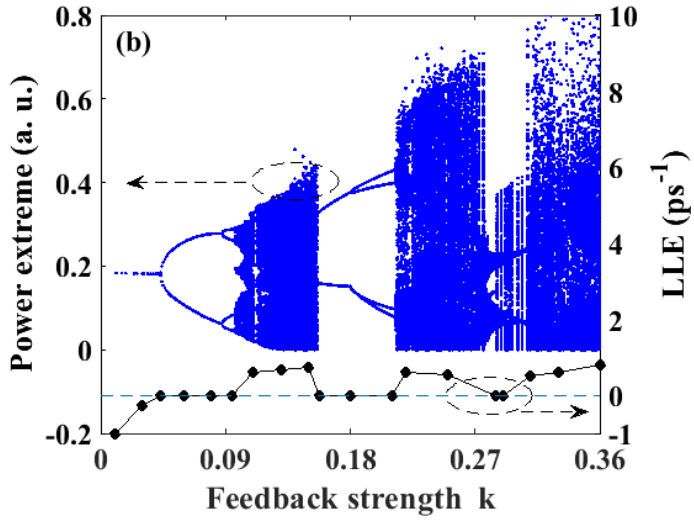

(b)

Figure 4. Bifurcation diagrams of power extreme and largest Lyapunov exponent (LLE) as a function of feedback strength of the GS lasing (a) and ES lasing (b) in a TSQDL biased at $120 \mathrm{~mA}$ under optical feedback with $\tau=100 \mathrm{ps}$.

Next, we discuss the influences of the round-trip time $(\tau)$ of the external cavity under a given feedback strength of $k=0.1$. Here, we only consider the case that $\tau$ is varied around $\tau_{0}=100 \mathrm{ps}$ within a very small range, in which the offset $(\Delta \tau)$ of $\tau$ from $\tau_{0}=100$ ps satisfies $-\pi / \omega_{G S} \leq \Delta \tau \leq \pi / \omega_{G S}$. Under this case, the phase offset $\varphi\left(=\Delta \tau \omega_{G S}\right)$ of GS lasing is varied within $(-\pi, \pi)$, and the corresponding phase offset of ES lasing is varied within $(-1.06 \pi, 1.06 \pi)$. Figure 5 presents the bifurcation diagrams of the power extreme and LLE of the GS lasing and ES lasing as a function of phase offset under $k=0.1$. With the increase of phase offset $\varphi$ from $-\pi$ to $\pi$, the dynamics evolution routes are more diverse. There exist multiple chaotic evolution routes for GS lasing and ES lasing including P1-S-C, P2-P1-P2-C, and C-MP-P2-C.

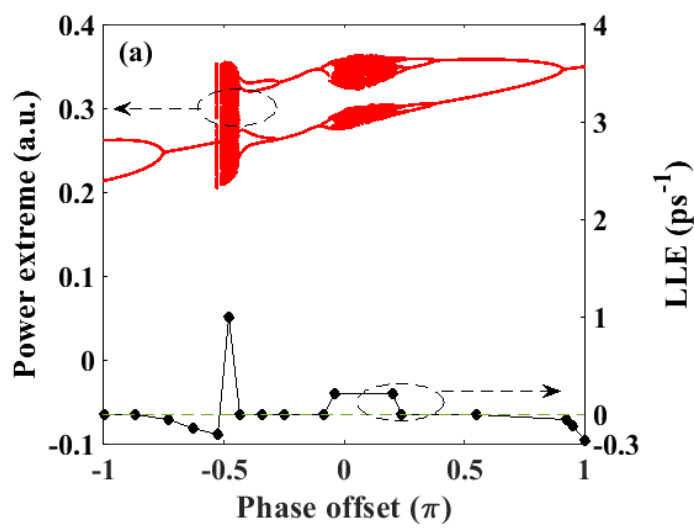

(a)

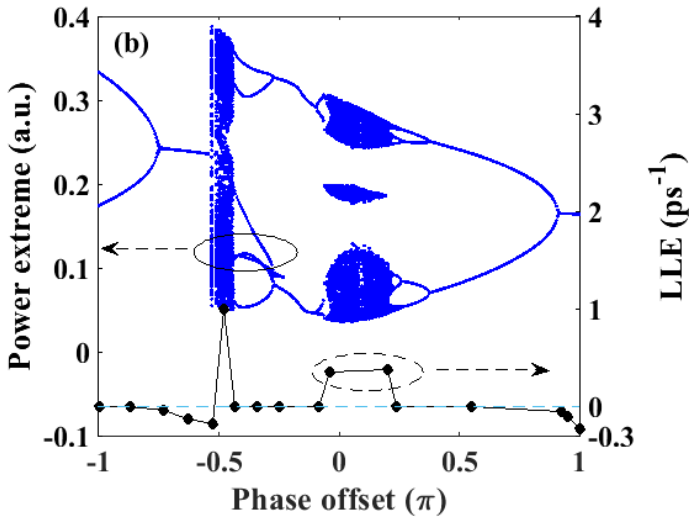

(b)

Figure 5. Bifurcation diagrams of the power extreme; LLE as a function of phase offset of the GS lasing (a) and ES lasing (b) in a TSQDL under $I=120 \mathrm{~mA}$ and $k=0.1$.

The above results demonstrate that the feedback strength and the round-trip time $\tau$ (equivalent to phase offset) of the external cavity are two crucial parameters affecting the nonlinear dynamics of TSQDLs. Therefore, it is essential to investigate the overall dynamical evolution in the parameter space of feedback strength and phase offset. Figure 6 presents the mapping of the dynamical states for GS lasing (a) and ES lasing (b) in the parameter space of feedback strength and phase offset. There are rich dynamic states including S, P1, P2, MP, and C in the parameter space. With the increase of feedback strength, the phase offset required for achieving a chaotic state is gradually widened. Although the dynamic state distributions of GS lasing and ES lasing are similar, there exist subtle differences at the boundary between two modes. Through observing this diagram 
carefully, it can be found that there are multiple evolution routes for driving the laser into the chaotic state such as S-P1-P2-MP-C, P1-P2-MP-C, and P1-MP-C.

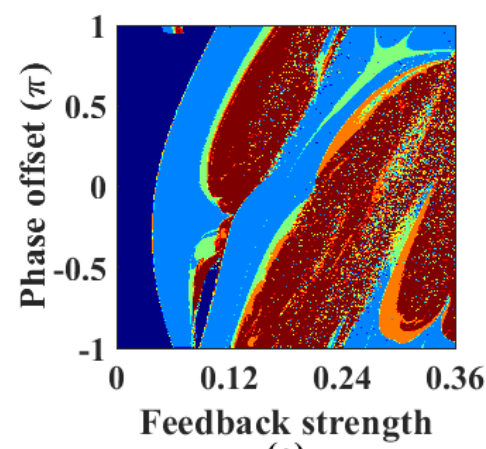

(a)

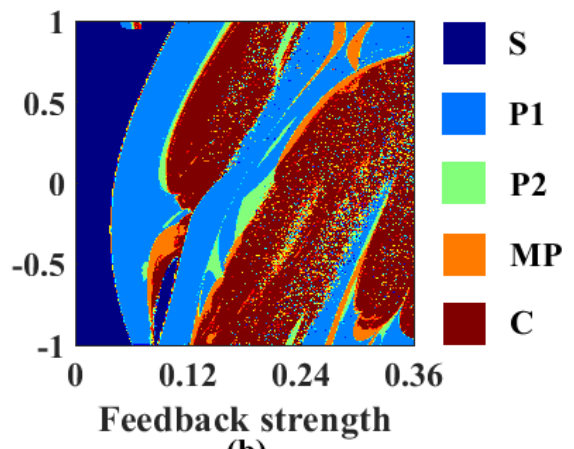

(b)

Figure 6. Mapping of the dynamical states for GS lasing (a) and ES lasing (b) of a TSQDL in the parameter space of feedback strength and phase offset. S: stable, P1: period-one, P2: period-two, MP: multi-period, and C: chaos.

Relevant research shows that the linewidth enhancement factor (LEF) $\alpha$ plays an important role for the nonlinear dynamics of SLs under external perturbations $[50,51]$. The above results were obtained under a fixed $\alpha$ taken as 3.5. Finally, we discuss the influences of LEF on the dynamical state distribution of a TSQDL under optical feedback. Figure 7 depicts mappings of dynamic states of GS lasing and ES lasing under different $\alpha$. For $\alpha=0.5$ (Figure 7(a1,a2), the dynamical states of GS and ES are relatively simple, which include S, P1, and C. In the whole parameter space, most of the region is in a stable state, and only a small region is in the chaotic state. For $\alpha=2.5$, as shown in Figure 7(b1,b2), there are much richer dynamic states involving P2 and MP. For a larger $\alpha$ of 4.5 as shown in Figure $7(\mathrm{c} 1, \mathrm{c} 2)$, the chaotic state occupies a large area. Therefore, a large $\alpha$ is helpful for achieving chaotic state output.

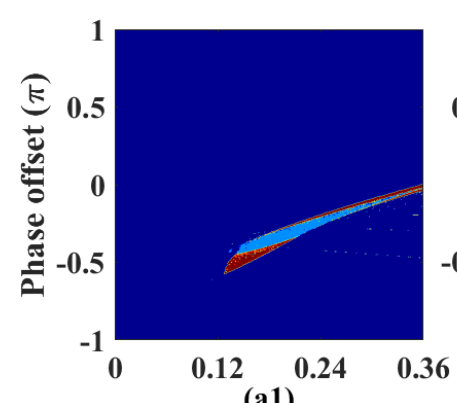

(a1)

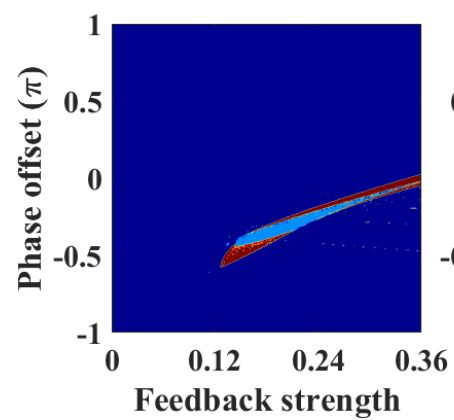

(a2)

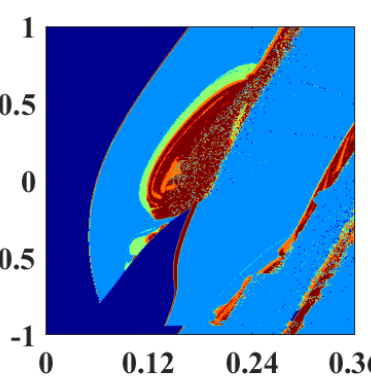

(b1)

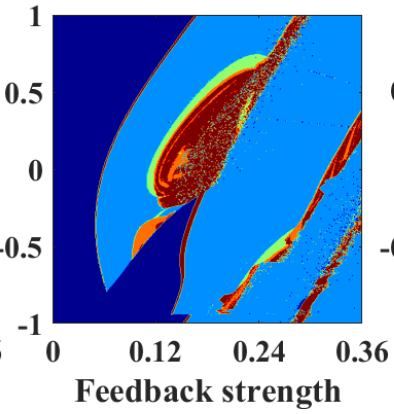

(b2)

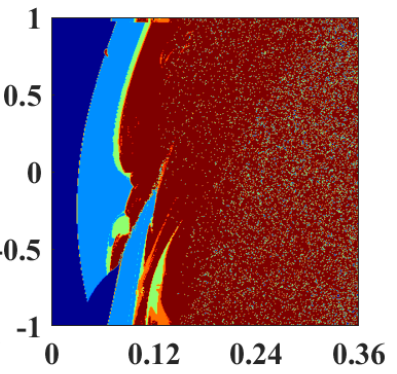

(c1)

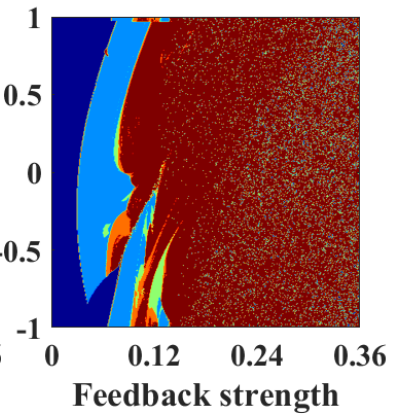

(c2)

Figure 7. Mappings of the dynamical states of GS lasing (the first row) and ES lasing (the second row) in the parameter space of feedback strength and phase offset under different $\alpha$, where (a) $\alpha=0.5,(\mathbf{b})$ $\alpha=2.5$, (c) $\alpha=4.5$. S: stable, P1: period-one, P2: period-two, MP: multi-period, and C: chaos. 
Additionally, it should be pointed out that above results were obtained under the condition that the spontaneous emission noises were ignored. In fact, after considering the influence of spontaneous emission noise, the boundary of dynamical states may be changed slightly.

\section{Conclusions}

In summary, via a rate equation model used to characterize TSQDLs with optical feedback, the nonlinear dynamics of TSQDLs subject to optical feedback were investigated theoretically. For a TSQDL biased at $120 \mathrm{~mA}$, both GS and ES lasing could be stimulated simultaneously, and the output power of GS emission was slightly larger than that of ES emission. After introducing optical feedback, multiple nonlinear dynamical states including S, P1, P2, MP, and C were observed for GS lasing and ES lasing under suitable feedback strengths and phase offset. Through mapping the evolution of dynamics state in the parameter space of feedback strength and phase offset, different evolution routes were revealed. In addition, the influences of the linewidth enhanced factor (LEF) on the dynamic state distribution of TSQDLs in the space parameter of feedback strength and phase shift were also presented. For a larger LEF, the parameter regions for GS lasing and ES lasing operating at chaotic state were wider. Although the dynamical behaviors of TSQDLs under optical feedback were similar to those observed in quantum well lasers under optical feedback, TSQDLs under optical feedback have the ability to provide two-channel chaotic signals with different lasing wavelengths, which are more promising for high-speed random number generation, wavelength-division multiplexing secure communication, and parallel-reservoir computing.

Author Contributions: X.-H.W. and Z.-F.J. were responsible for the numerical simulation, analyzing the results, and the writing of the paper. Z.-M.W. and G.-Q.X. were responsible for the discussion of the results and reviewing/editing/revising/proof-reading of the manuscript. All authors have read and agreed to the published version of the manuscript.

Funding: This research was funded by the National Natural Science Foundation of China (Grant Nos. 61775184 and 61875167).

Data Availability Statement: Not applicable.

Conflicts of Interest: The authors declare no conflict of interest.

\section{References}

1. Lin, F.Y.; Tu, S.Y.; Huang, C.C.; Chang, S.M. Nonlinear dynamics of semiconductor lasers under repetitive optical pulse injection. IEEE J. Sel. Top. Quantum Electron. 2009, 15, 604-611. [CrossRef]

2. Yuan, G.H.; Yu, S.Y. Bistability and switching properties of semiconductor ring lasers with external optical injection. IEEE J. Quantum Electron. 2008, 44, 41-48. [CrossRef]

3. Kawaguchi, Y.; Okuma, T.; Kanno, K.; Uchida, A. Entropy rate of chaos in an optically injected semiconductor laser for physical random number generation. Opt. Express 2021, 29, 2442-2457. [CrossRef] [PubMed]

4. Jiang, N.; Xue, C.P.; Lv, Y.X.; Qiu, K. Physically enhanced secure wavelength division multiplexing chaos communication using multimode semiconductor lasers. Nonlinear Dyn. 2016, 86, 1937-1949. [CrossRef]

5. Li, N.Q.; Pan, W.; Xiang, S.Y.; Yan, L.S.; Luo, B.; Zou, X.H.; Zhang, L.Y.; Mu, P.H. Photonic generation of wideband timedelay-signature-eliminated chaotic signals utilizing an optically injected semiconductor laser. IEEE J. Quantum Electron. 2012, 48, 1339-1345. [CrossRef]

6. Salvide, M.F.; Masoller, C.; Torre, M.S. All-optical stochastic logic gate based on a VCSEL with tunable optical injection. IEEE J. Quantum Electron. 2013, 49, 886-893. [CrossRef]

7. Guo, X.X.; Xiang, S.Y.; Zhang, Y.H.; Lin, L.; Wen, A.J.; Hao, Y. Polarization multiplexing reservoir computing based on a VCSEL with polarized optical feedback. IEEE J. Sel. Top. Quantum Electron. 2020, 26, 1700109. [CrossRef]

8. Liu, G.T.; Stintz, A.; Li, H.; Malloy, K.J.; Lester, L.F. Extremely low room-temperature threshold current density diode lasers using InAs dots in $\operatorname{In}_{0.15} \mathrm{Ga}_{0.85}$ As quantum well. Electron. Lett. 1999, 35, 1163-1165. [CrossRef]

9. Mikhrin, S.S.; Kovsh, A.R.; Krestnikov, I.L.; Kozhukhov, A.V.; Livshits, D.A.; Ledentsov, N.N.; Shernyakov, Y.M.; Novikov, I.I.; Maximov, M.V.; Ustinov, V.M.; et al. High power temperature-insensitive $1.3 \mu \mathrm{m}$ InAs/InGaAs/GaAs quantum dot lasers. Semicond. Sci. Technol. 2005, 20, 340-342. [CrossRef] 
10. Saito, H.; Nishi, K.; Kamei, A.; Sugou, S. Low chirp observed in directly modulated quantum dot lasers. IEEE Photonics Technol. Lett. 2000, 12, 1298-1300. [CrossRef]

11. Kuntz, M.; Fiol, G.; Lammlin, M.; Schubert, C.; Kovsh, A.R.; Jacob, A.; Umbach, A.; Bimberg, D. 10Gb/s data modulation using $1.3 \mu \mathrm{m}$ InGaAs quantum dot lasers. Electron. Lett. 2005, 41, 244-245. [CrossRef]

12. Norman, J.C.; Jung, D.; Zhang, Z.Y.; Wan, Y.T.; Liu, S.T.; Shang, C.; Herrick, R.W.; Chow, W.W.; Gossard, A.C.; Bowers, J.E. A review of high-performance quantum dot lasers on silicon. IEEE J. Quantum Electron. 2019, 55, 2000511. [CrossRef]

13. Norman, J.C.; Jung, D.; Wan, Y.T.; Bowers, J.E. Perspective: The future of quantum dot photonic integrated circuits. APL Photonics 2018, 3, 030901. [CrossRef]

14. Zhukov, A.E.; Kovsh, A.R. Quantum dot diode lasers for optical communication systems. Quantum Electron. 2008, 38, 409-423. [CrossRef]

15. Wang, C.; Raghunathan, R.; Schires, K.; Chan, S.C.; Lester, L.F.; Grillot, F. Optically injected InAs/GaAs quantum dot laser for tunable photonic microwave generation. Opt. Lett. 2016, 41, 1153-1156. [CrossRef]

16. Jiang, Z.F.; Wu, Z.M.; Yang, W.Y.; Hu, C.X.; Lin, X.D.; Jin, Y.H.; Dai, M.; Cui, B.; Yue, D.Z.; Xia, G.Q. Numerical simulations on narrow-linewidth photonic microwave generation based on a QD laser simultaneously subject to optical injection and optical feedback. Appl. Opt. 2020, 59, 2935-2941. [CrossRef]

17. Viktorov, E.A.; Mandel, P.; Tanguy, Y.; Houlihan, J.; Huyet, G. Electron-hole asymmetry and two-state lasing in quantum dot lasers. Appl. Phys. Lett. 2005, 87, 053113. [CrossRef]

18. Meinecke, S.; Lingnau, B.; Rohm, A.; Ludge, K. Stability of optically injected two-state quantum-dot lasers. Ann. Phys. 2017, 529, 1600279. [CrossRef]

19. Li, Q.Z.; Wang, X.; Zhang, Z.Y.; Chen, H.M.; Huang, Y.Q.; Hou, C.C.; Wang, J.; Zhang, R.Y.; Ning, J.Q.; Min, J.H.; et al. Development of modulation p-doped $1310 \mathrm{~nm}$ InAs/GaAs quantum dot laser materials and ultrashort cavity Fabry-Perot and distributed-feedback laser diodes. ACS Photonics 2018, 5, 1084-1093. [CrossRef]

20. Grillot, F.; Norman, J.C.; Duan, J.; Zhang, Z.; Dong, B.; Huang, H.; Chow, W.W.; Bowers, J.E. Physics and applications of quantum dot lasers for silicon photonics. J. Nanophotonics 2020, 9, 1271-1286. [CrossRef]

21. O’Brien, D.; Hegarty, S.P.; Huyet, G.; McInerney, J.G.; Kettler, T.; Laemmlin, M.; Bimberg, D.; Ustinov, V.M.; Zhukov, A.E.; Mikhrin, S.S.; et al. Feedback sensitivity of $1.3 \mathrm{mu} \mathrm{m}$ InAs/GaAs quantum dot lasers. Electron. Lett. 2003, 39, 1819-1820. [CrossRef]

22. Huyet, G.; O’Brien, D.; Hegarty, S.P.; McInerney, J.G.; Uskov, A.V.; Bimberg, D.; Ribbat, C.; Ustinov, V.M.; Zhukov, A.E.; Mikhrin, S.S.; et al. Quantum dot semiconductor lasers with optical feedback. Phys. Status Solidi A 2003, 201, 345-352. [CrossRef]

23. Stevens, B.J.; Childs, D.T.D.; Shahid, H.; Hogg, R.A. Direct modulation of excited state quantum dot lasers. Appl. Phys. Lett. 2009, 95, 061101. [CrossRef]

24. Lee, C.S.; Bhattacharya, P.; Frost, T.; Guo, W. Characteristics of a high speed $1.22 \mu \mathrm{m}$ tunnel injection p-doped quantum dot excited state laser. Appl. Phys. Lett. 2011, 98, 011103. [CrossRef]

25. Lin, L.C.; Chen, C.Y.; Huang, H.M.; Arsenijevic, D.; Bimberg, D.; Grillot, F.; Lin, F.Y. Comparison of optical feedback dynamics of InAs/GaAs quantum-dot lasers emitting solely on ground or excited states. Opt. Lett. 2018, 43, 210-213. [CrossRef] [PubMed]

26. Jiang, Z.F.; Wu, Z.M.; Jayaprasath, E.; Yang, W.Y.; Hu, C.X.; Xia, G.Q. Nonlinear dynamics of exclusive excited-state emission quantum dot lasers under optical injection. Photonics 2019, 6, 58. [CrossRef]

27. Xu, P.F.; Yang, T.; Ji, H.M.; Cao, Y.L.; Gu, Y.X.; Liu, Y.; Ma, W.Q.; Wang, Z.G. Temperature-dependent modulation characteristics for $1.3 \mu \mathrm{m}$ InAs/GaAs quantum dot lasers. J. Appl. Phys. 2010, 107, 013102.

28. Pawlus, R.; Columbo, L.L.; Bardella, P.; Breuer, S.; Gioannini, M. Intensity noise behavior of an InAs/InGaAs quantum dot laser emitting on ground states and excited states. Opt. Lett. 2018, 43, 867-870. [CrossRef] [PubMed]

29. Naderi, N.A.; Grillot, F.; Yang, K.; Wright, J.B.; Gin, A.; Lester, L.F. Two-color multi-section quantum dot distributed feedback laser. Opt. Express 2010, 18, 27028-27035. [CrossRef] [PubMed]

30. Cataluna, M.A.; Nikitichev, D.I.; Mikroulis, S.; Simos, H.; Simos, C.; Mesaritakis, C.; Syvridis, D.; Krestnikov, I.; Livshits, D.; Rafailov, E.U. Dual-wavelength mode-locked quantum-dot laser, via ground and excited state transitions: Experimental and theoretical investigation. Opt. Express 2012, 18, 12832-12838. [CrossRef]

31. Breuer, S.; Rossetti, M.; Drzewietzki, L.; Bardella, P.; Montrosset, I.; Elsasser, W. Joint experimental and theoretical investigations of two state mode locking in a strongly chirped reverse biased monolithic quantum dot laser. IEEE J. Quantum Electron. 2011, 47, 1320-1329. [CrossRef]

32. Mesaritakis, C.; Kapsalis, A.; Bogris, A.; Syvridis, D. Artificial neuron based on integrated semiconductor quantum dot modelocked lasers. Sci. Rep. 2016, 6, 39317. [CrossRef] [PubMed]

33. Tykalewicz, B.; Goulding, D.; Hegarty, S.P.; Huyet, G.; Byrne, D.; Phelan, R.; Kelleher, B. All-optical switching with a dual-state, single-section quantum dot laser via optical injection. Opt. Lett. 2014, 39, 4607-4610. [CrossRef]

34. Viktorov, E.A.; Dubinkin, I.; Fedorov, N.; Erneux, T.; Tykalewicz, B.; Hegarty, S.P.; Huyet, G.; Goulding, D.; Kelleher, B. Injection-induced, tunable, all-optical gating in a two-state quantum dot laser. Opt. Lett. 2016, 41, 3555-3558. [CrossRef]

35. Tykalewicz, B.; Goulding, D.; Hegarty, S.P.; Huyet, G.; Dubinkin, I.; Fedorov, N.; Erneux, T.; Viktorov, E.A.; Kelleher, B. Optically induced hysteresis in a two-state quantum dot laser. Opt. Lett. 2016, 41, 1034-1037. [CrossRef] [PubMed]

36. Jiang, Z.F.; Wu, Z.M.; Jayaprasath, E.; Yang, W.Y.; Hu, C.X.; Cui, B.; Xia, G.Q. Power-induced lasing state switching and bistability in a two-state quantum dot laser subject to optical injection. Opt. Appl. 2020, 50, 257-269. 
37. Virte, M.; Breuer, S.; Sciamanna, M.; Panajotov, K. Switching between ground and excited states by optical feedback in a quantum dot laser diode. Appl. Phys. Lett. 2014, 105, 121109. [CrossRef]

38. Virte, M.; Panajotov, K.; Sciamanna, M. Mode competition induced by optical feedback in two-color quantum dot lasers. IEEE J. Quantum Electron. 2013, 49, 578-585. [CrossRef]

39. Virte, M.; Pawlus, R.; Sciamanna, M.; Panajotov, K.; Breuer, S. Energy exchange between modes in a multimode two-color quantum dot laser with optical feedback. Opt. Lett. 2016, 41, 3205-3208. [CrossRef]

40. Meinecke, S.; Kluge, L.; Hausen, J.; Lingnau, B.; Ludge, K. Optical feedback induced oscillation bursts in two-state quantum-dot lasers. Opt. Express 2020, 28, 3361-3377. [CrossRef]

41. Viktorov, E.A.; Mandel, P.; O’Driscoll, I.; Carroll, O.; Huyet, G.; Houlihan, J.; Tanguy, Y. Low-frequency fluctuations in two-state quantum dot lasers. Opt. Lett. 2006, 31, 2302-2304. [CrossRef]

42. Zhou, Y.G.; Duan, J.N.; Grillot, F.; Wang, C. Optical noise of dual-state lasing quantum dot lasers. IEEE J. Quantum Electron. 2020, 56, 2001207. [CrossRef]

43. Veselinov, K.; Grillot, F.; Cornet, C.; Even, J.; Bekiarski, A.; Gioannini, M.; Loualiche, S. Analysis of the double laser emission occurring in 1.55-mu m InAs-InP (113) B quantum-dot lasers. IEEE J. Quantum Electron. 2007, 43, 810-816. [CrossRef]

44. Dehghaninejad, A.; Sheikhey, M.M.; Baghban, H. Dynamic behavior of injection-locked two-state quantum dot lasers. J. Opt. Soc. Am. B 2019, 36, 1518-1524. [CrossRef]

45. Wang, C.; Grillot, F.; Even, J. Impacts of wetting layer and excited state on the modulation response of quantum-dot lasers. IEEE J. Quantum Electron. 2012, 48, 1144-1150. [CrossRef]

46. Ohnesorge, B.; Albrecht, M.; Oshinowo, J.; Forchel, A.; Arakawa, Y. Rapid carrier relaxation in self-assembled InxGa1-xAs/GaAs quantum dots. Phys. Rev. B 1996, 54, 11532-11538. [CrossRef]

47. Ignatiev, I.V.; Kozin, I.E.; Nair, S.V.; Ren, H.W.; Sugou, S.; Masumoto, Y. Carrier relaxation dynamics in InP quantum dots studied by artificial control of nonradiative losses. Phys. Rev. B 2000, 61, 15633-15636. [CrossRef]

48. Grillot, F.; Wang, C.; Nederi, N.A.; Even, J. Modulation Properties of Self-Injected Quantum-Dot Semiconductor Diode Lasers. IEEE J. Sel. Top. Quantum Electron. 2013, 19, 1900812. [CrossRef]

49. Chen, H.; Lei, T.F.; Lu, S.; Dai, W.P.; Qiu, L.J.; Zhong, L. Dynamics and Complexity Analysis of Fractional-Order Chaotic Systems with Line Equilibrium Based on Adomian Decomposition. Complexity 2020, 2020, 5710765. [CrossRef]

50. AL-Hosiny, N.M. Effect of linewidth enhancement factor on the stability map of optically injected distributed feedback laser. Opt. Rev. 2014, 21, 261-264. [CrossRef]

51. Abdulrhmann, S.; Yamada, M.; Ahmed, M. Numerical modeling of the output and operations of semiconductor lasers subject to strong optical feedback and its dependence on the linewidth-enhancement factor. Int. J. Numer. Model. Electron. Netw. Devices Fields 2011, 24, 218-229. [CrossRef] 